\title{
Larger Sperm Size may Contribute to Reproductive Isolation between Etheostoma Species
}

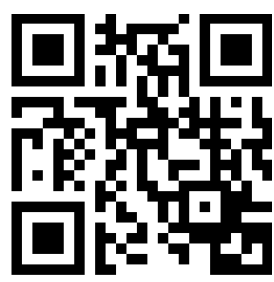

\author{
Mercy Arkorful ${ }^{1}$, Katrina L. Gazo ${ }^{1}$, Aaron Zweig ${ }^{2}$, Laura E. Ott ${ }^{3}$, Tamra Mendelson ${ }^{4}$, Tagide \\ deCarvalho ${ }^{3}$
}

Etheostoma is a genus of North American darter fish whose species have similar habitats and breeding seasons, yet hybridization is rare. Behavioral barriers have been demonstrated to play a key role in maintaining species boundaries. Further, conspecific (same species) sperm precedence has also been observed when the gametes of two different species come into contact. In this study, we investigated if physical characteristics of sperm could be a mechanism for the lower fertilization success of heterospecific (different species) males when eggs are simultaneously exposed to conspecific and heterospecific sperm. We chose to examine the sperm of two closely related species, E. zonale and E. barrenense. Using toluidine blue and immunofluorescent labeling methods, we compared head diameter and tail length of sperm cells between the two species. We found that head diameter was significantly larger for E. barrenense sperm compared to E. zonale. This difference in cell morphology may point to a physical mechanism underlying conspecific sperm precedence in Etheostoma. Our results are the first to describe a morphological difference in sperm between species in this genus and provide initial evidence for the role of sperm morphology in prezygotic reproductive isolation.

\section{INTRODUCTION}

Etheostoma, commonly known as darter fish, provide an excellent system to study the evolution of reproductive isolation (Williams \& Mendelson, 2010). Isolating mechanisms in these fish are intriguing, since they live in similar habitats, have similar breeding seasons, and fertilize externally. Therefore, multiple forms of reproductive isolation must be at work to prevent these species from hybridizing, given that there are ample opportunities for gametes to mix. It has been determined that behavioral pre-mating isolation is very strong and nearly complete in these fish (Mendelson et al., 2007). However, this does not address what prevents hybridiza-

\footnotetext{
${ }^{1}$ Howard Community College, 10901 Little Patuxent Pkwy, Columbia, MD 21044

${ }^{2}$ Montgomery College, 51 Mannakee St, Rockville, MD 20850

${ }^{3}$ College of Natural and Mathematical Sciences, University of Maryland, Baltimore County, 1000 Hilltop Cir, Baltimore, MD 21250
}

${ }^{4}$ Department of Biological Sciences, University of Maryland, Baltimore County, 1000 Hilltop Cir, Baltimore, MD 21250

*To whom correspondence should be addressed: leott@umbc.edu

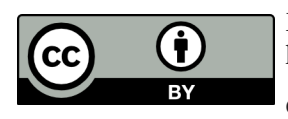

Except where otherwise noted, this work is licensed under https://creativecommons.org/licenses/by/4.0

doi:10.22186/jyi.35.6.92-96 tion after multiple species spawn in the same locality. Barriers to hybridization that occur after mating are referred to as forms of post-mating prezygotic reproductive isolation.

This type of isolation occurs when similar species can mate or spawn but are unable to cross-fertilize and successfully produce hybrids. Gametic incompatibility between species often contributes to post-mating prezygotic reproductive isolation (Lessios \& Cunningham, 1990). Gametic incompatibility is not a barrier to reproduction in Etheostoma, but conspecific sperm precedence has been demonstrated in the laboratory (Mendelson et al., 2007; Williams \& Mendelson, 2014). This suggests that there is an aspect to sperm physiology or structure that gives conspecifics an advantage for fertilization in the presence of another species' sperm.

Sperm consist of three distinct regions: head, midpiece, and tail. Despite this basic structure, sperm morphology is incredibly varied across the animal kingdom. Heads differ in shape and size, midpieces vary in volume, and tails are diverse in length between species (Islam \& Akhter, 2012; Anderson et al., 2005). LaMunyon and Ward (2002) suggest that variance in sperm morphology between species may be a consequence of the evolutionary process of sperm competition within a species, where sperm competition is defined as two or more conspecific males vying for the same egg (Parker, 1970).

Research on sperm competition within species has shown that sperm with increased head diameter, tail length, overall size, and midpiece volume are more successful at fertilization (Anderson et al., 2005; Byrne et al., 2003; Gomendio \& Roldan, 1991). LaMunyon and Ward (2002) also suggest that sperm size differences across species may also be the result of sperm competition; when 

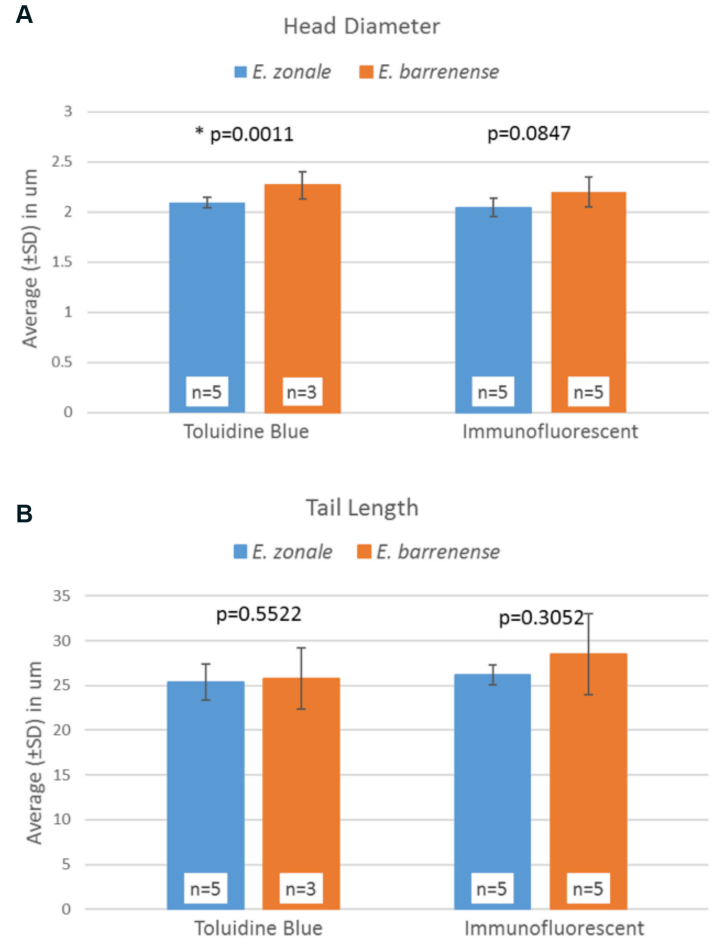

Figure 1. Comparison of E. zonale and E. barrenense Head Diameters (A) and Tail Length (B). Sperm from both species were stained using toluidine blue and immunofluorescent labeling techniques. Data depicted is average \pm standard deviation (SD) and $n$ indicates the number of individual fish evaluated.

sperm competition was increased experimentally within different worm species, sperm size also increased. Thus, if sperm size differences can affect fertilization success within a species, it may also contribute to sperm precedence or even gametic incompatibility between species. Therefore, we aimed to investigate patterns in sperm size in the context of reproductive isolation instead of sperm competition. To identify a potential mechanism for conspecific sperm precedence, we hypothesized that closely-related species would display variation in sperm dimensions. We used two species of Etheostoma that occupy the same habitat and thus spawn in the same geographic location to address this hypothesis.

The purpose of this exploratory study was to investigate physical variations in the sperm of $E$. barrenense and E. zonale as a potential mechanism of reproductive isolation between these two species. Previous studies on these and other Etheostoma have already demonstrated that sperm from other species do not have the same fertilization success as conspecific sperm, but the mechanism of gametic incompatibility is unknown (Mendelson et al., 2007; Williams \& Mendelson, 2014). This research is the first of its kind to analyze possible differences in sperm size as it relates to reproductive isolation in fish, as prior studies on morphological differences in sperm as a reproductive barrier have been limited to sea urchins (Hudson et al., 2015; Landry et a,. 2003; Marks et al., 2008). We used histological and immunofluorescent labeling tech- niques to measure different aspects of head size, including capsule and nucleus diameter, and we used both techniques to measure tail length.

\section{MATERIALS AND METHODS}

Fish were harvested from the East Fork of the Barren River, Monroe Co., KY in the spring of 2016. Fish were anesthetized by immersion in $0.004 \%$ MS-222 (tricaine) and then placed on a petri dish for sperm collection. Ejaculate was expressed via pressure around the urogenital region and immediately fixed with a $4 \%$ formaldehyde (in PBS) solution. Individual male samples were saved in separate vials and stored at $4{ }^{\circ} \mathrm{C}$. The UMBC Institutional Animal Care and Use Committee (IACUC) approved all protocols involving ejaculate collection. $10 \mu \mathrm{L}$ of each male's sample was pipetted onto a silanated slide and let dry, then washed with phosphate buffered saline (PBS). Toluidine blue solution (1\% Toluidine blue and $1 \%$ sodium borate) was used to measure head capsule and tail length by placing the dye on the sample for 5 minutes before washing with PBS. Samples were mounted with glycerol and sealed beneath coverslips with clear nail polish.

Fluorescent staining was used to measure head nucleus size, as well as tail length. Anti-alpha-tubulin (bovine) mouse IgG1 monoclonal primary antibody (Life Technologies, Eugene, OR) was used to stain tubulin proteins abundant in sperm flagellum. 50 $\mu \mathrm{L}$ of a $1: 250$ dilution in $1 \%$ bovine serum albumin (BSA) in PBS was applied to the samples and incubated in a moisture box at $4{ }^{\circ} \mathrm{C}$ overnight. Alexa Fluor 488 goat anti-mouse IgG (Life Technologies) was used to label the primary antibody. $50 \mu \mathrm{L}$ of a 1:500 dilution in PBS was added to the samples and incubated in a moisture box for 30 minutes at room temperature. The fluorescent molecule 4',6-diamidino-2-phenylindole (DAPI) was used to stain nucleic acid (Tarnowski, Spinale, \& Nicholson, 1991). $30 \mu \mathrm{L}$ of $300 \mathrm{nM}$ DAPI was placed on the sample and incubated in a covered moisture box at room temperature for 30 minutes.

Photomicrographs of sperm dyed with toluidine blue were taken using the Zeiss Primo Star HAL Microscope using brightfield illumination with a $100 \mathrm{x}$ objective (total magnification $=$ 1000x). Measurements were taken using ImageJ for Windows (64bit). Head diameter was measured at its widest point perpendicular to the tail. Fluorescent images were taken using a Leica SP5 confocal microscope. The nucleus diameter was measured at the widest point perpendicular to the tail in the DAPI channel. Tail length was determined using a combination of the GFP channel, bright field, and composite images.

Averages and standard deviations of morphological measurements were calculated using Microsoft Excel 2010. Multiple sperm cells were measured from each individual male sample. The average head and tail values for each male was used for specieslevel comparisons. Toluidine blue labeled sperm measurements came from 5 E. zonale males $(n=30$ sperm cells) and $3 E$. barrenense males ( $n=7$ sperm cells). Fluorescently-labeled sperm measurements came from 5 E. zonale males ( $n=50$ sperm cells) and 5 E. barrenense males ( $n=40$ sperm cells). Statistical analysis 


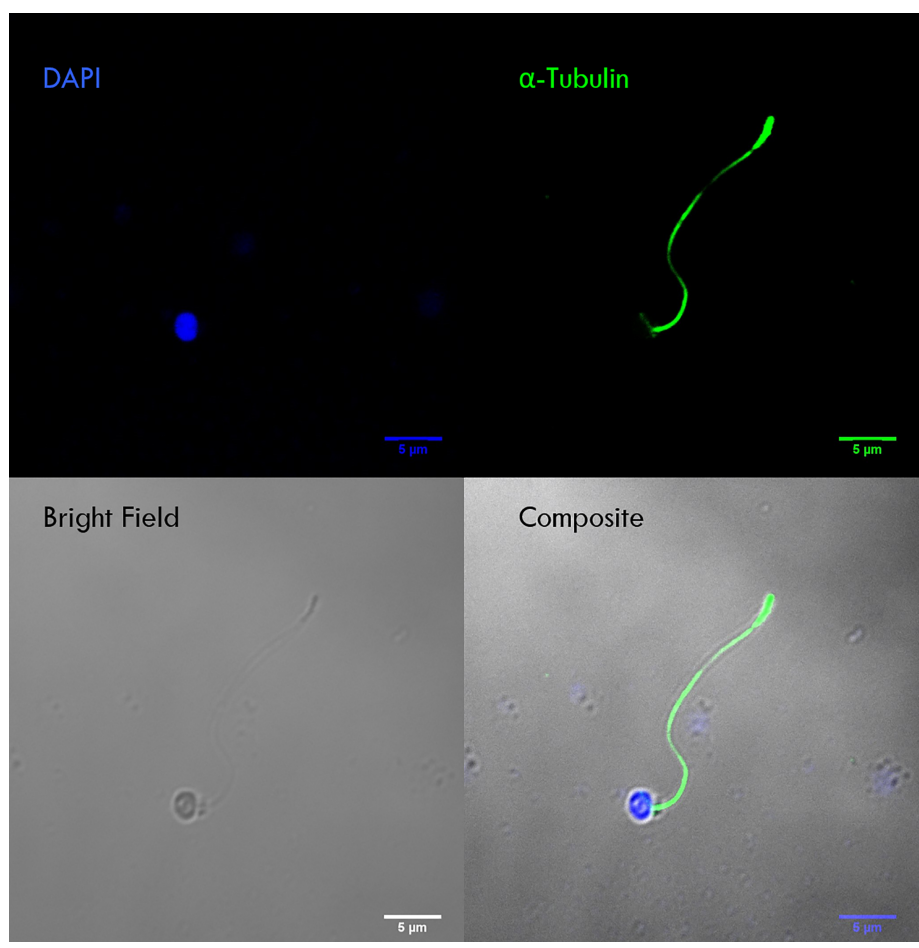

Figure 2. Fluorescently-labeled $\boldsymbol{E}$. barrenense sperm cell. The nucleus can be identified by DAPI staining (upper left) and the tail can be identified by the alpha-tubulin Alexa 488 staining (upper right). The bottom right is the composite; the bottom left shows a bright field image.

was performed using a 2-sample t-test $(\alpha=0.05)$ and StatCrunch online software.

\section{RESULTS}

For toluidine blue staining, E. barrenense sperm had an average head diameter of $2.27( \pm 0.13) \mu \mathrm{m}$ and tail length of $25.75( \pm 3.42)$ $\mu \mathrm{m}$ (Figure 1). Fluorescently labeled E. barrenense sperm yielded an average nuclear diameter of $2.20( \pm .15) \mu \mathrm{m}$ and tail length of $28.52( \pm 4.52) \mu \mathrm{m}$ (see Figure 2 for representative image). E. zonale stained with toluidine blue had an average head diameter of $2.10( \pm 0.05) \mu \mathrm{m}$ and tail length of $25.39( \pm 1.97) \mu \mathrm{m}$. The average nuclear diameter for $E$. zonale samples stained with immunofluorescent labeling were $2.04( \pm 0.09) \mu \mathrm{m}$ and tail lengths of $26.18( \pm$ 1.09) $\mu \mathrm{m}$ (Figure 1). E. barrenense sperm were shown to have a statistically significant larger head capsule diameter $(p=0.0011)$, on average, compared to E. zonale when stained with toluidine blue. Nuclear diameter, as in the DAPI staining for fluorescent labeling, showed no significant differences between the two species $(p=0.0847)$. Tail length did not differ statistically between the species from either labeling approach (Figure 1B).

\section{DISCUSSION}

In this exploratory study, we investigated the morphology of sperm from two darter species, E. zonale and E. barrenense, to examine variation that might lead to sperm precedence previously described for these and other darter species (Mendelson et al., 2007;
Williams \& Mendelson, 2014). Barriers to successful fertilization between species can arise from either chemical or morphological features of sperm (Byrne et al., 2003; Landry et al., 2003; Mendelson et al., 2007). Head size (i.e., diameter) and tail length are often used to compare morphological variation in sperm among species (Gage \& Cook, 1994). We therefore focused on these morphological characteristics as these measurements are simple to take and convey robust data concerning sperm cell morphology.

We discovered that $E$. barrenense has a larger sperm head than $E$. zonale. Although we were limited by the small number of males used in this study, a significant difference $(p=0.0011)$ in sperm head diameter was still observed. Variation in sperm head width is common among fish species; however, the functional significance is still unknown (Jamieson, 1991). Montoto et al. (2011) hypothesized that in rodents, relatively larger heads may block smaller rival sperm from simultaneously attaching to an egg. Applying similar reasoning to Etheostoma, we would expect that because $E$. barrenense heads are relatively larger, a relatively low number of hybrids would form between $E$. barrenense females and $E$. zonale males. However, Williams \& Mendelson (2014) performed these crosses for conspecific sperm precedence and found the opposite pattern. To further test if head size is a factor in conspecific sperm precedence, individual E. zonale males with sperm size differences could be examined in a competitive cross (Williams \& Mendelson, 2014). If head size is a factor for conspecific sperm precedence, we would anticipate that ejaculate from individual males with relatively smaller sperm would have greater fertilization success.

In addition, we were interested in exploring whether the difference in head size between species was due to direct selection on the sperm capsule. In fish, the sperm head consists mainly of the nucleus (Jamieson, 1991) and nuclear size has a strong correlation with genome size (Beaulieu et al., 2008; Gregory, 2001). Thus, differences in darter sperm head size could merely be a by-product of differences in genome size. Alternatively, if there was direct selection for sperm head size to reduce interspecific hybridization or increase sperm competition success, we would expect to see a difference in sperm capsule but not nuclear size. We compared nuclear diameters between the two species and found no significant difference. This supports the hypothesis that there may have been direct selection on sperm head size, which further promotes the idea that sperm size differences may be functionally relevant.

To examine potential interspecific differences in tail length, we used measurements from both toluidine blue and fluorescently labeled sperm. We found that the tails of E. zonale and E. barrenense sperm were not significantly different in length. This was an unexpected result because sperm tail length is often highly variable between closely related species, including fish (Jamieson, 1991) and tail length often plays a role in the sperm competition success (Byrne et al., 2003; Gomendio \& Roldan, 1991).

The results of this exploratory study suggest that differences in Etheostoma sperm cell morphology may play a role in the hybridization barriers that exist within this genus. Our data demonstrated a robust difference in sperm head diameter between $E$. zonale and 
E. barrenense. Furthermore, the size difference reflects the size of the head capsule and not the nucleus, suggesting that there has been direct selection on sperm head size. However, we cannot say conclusively if there is no interspecific difference in nuclear size due to our small sample size. We were limited by the number of males that were able to produce ejaculate and a one-time collection of ejaculate, because captive males were at the end of their reproductive season when we began the study. An improved sample size would help answer this question. However, if increased data demonstrate that nuclear size is driving head size, this does not rule out that size differences play a role in conspecific sperm precedence.

Although an increase in sperm head size has already been shown to have increased success in fertilization (Yániz et al., 2015), these studies focus on animals with internal fertilization. Sperm precedence is likely to be an even more important barrier to hybridization for external fertilization because gametes mingle freely in water without the additional anatomical barriers present with internal fertilization. Yet little work has been done to examine interspecific differences in sperm morphology in this context. Similar to this study, differences in sperm head size of closely related sea urchins have been postulated as a mechanism of reproductive isolation (Hudson et al., 2015; Landry et al., 2003; Marks et al., 2008). However, future work in darters and other animals with external fertilization needs to focus on whether variation in sperm head size translates into differences in fertilization success..

\section{ACKNOWLEDGMENTS}

The authors would like to thank the STEM BUILD at UMBC Initiative for their support of this project. The authors would also like to thank Ryan Buckley for his technical assistance. Shreya Agarwal, Jerson Guansing, Minhquan Tran, and Chloe Kwon were invaluable for their practical advice and on-hand mentorship. Use of the confocal microscope was made possible by the NSF MRI Grant DBI-0722568. The STEM BUILD at UMBC Initiative is funded by the National Institute of General Medical Sciences (NIH grant numbers 8TL4GM118989, 8UL1GM118988, and 8RL5GM118987).

\section{REFERENCES}

Anderson, M. J., Nyholt, J., \& Dixson, A. F. (2005). Sperm Competition and the Evolution of Sperm Midpiece Volume in Mammals. Journal of Zoology, 267(02), 135-142. doi:10.1017/s0952836905007284

AntiBovine $\alpha$-Tubulin, Mouse Monoclonal Antibodies. (2001). Molecular Probes, Inc. Retrieved from https://tools.thermofisher.com/content/sfs/manuals/ mp11126.pdf

Beaulieu, J. M., Leitch, I. J., Patel, S., Pendharkar, A., \& Knight, C. A. (2008). Genome Size Is a Strong Predictor of Cell Size and Stomatal Density in Angiosperms. New Phytologist, 179(4), 975-986. doi:10.1111/j.14698137.2008.02528.x

Byrne, P. G., Simmons, L. W., \& Roberts, J. D. (2003). Sperm Competition and the Evolution of Gamete Morphology in Frogs. Proceedings of the Royal Society B: Biological Sciences, 270(1528), 2079-2086. doi:10.1098/ rspb.2003.2433

DAPI Protocol for Fluorescence Imaging. (n.d.). Retrieved June 21, 2016, from https://www.thermofisher.com/us/en/home/references/protocols/cell-andtissue-analysis/protocols/dapi-imaging-protocol.html

Electron Microscopy Sciences. Glycerol Mounting Medium. Hatfield, PA.
Esteso, M. C., Soler, A. J., Fernandez-Santos, M. R., Quintero-Moreno, A. A., \& Garde, J. J. (2006). Functional Significance of the Sperm Head Morphometric Size and Shape for Determining Freezability in Iberian Red Deer (Cervus elaphus hispanicus) Epididymal Sperm Samples. Journal of Andrology, 27(5), 662-670. doi:10.2164/jandrol.106.000489

Fiji [Computer software]. (2016). Retrieved from http://imagej.net/Fiji/Downloads. Gage, M. J., \& Cook, P. A. (1994). Sperm Size or Numbers? Effects of Nutritional Stress Upon Eupyrene and Apyrene Sperm Production Strategies in the Moth Plodia interpunctella (Lepidoptera: Pyralidea). Functional Ecology, 8(5), 594-599. doi:10.2307/2389920

Gomendio, M., \& Roldan, E. R. (1991). Sperm Competition Influences Sperm Size in Mammals. Proceedings of the Royal Society B: Biological Sciences, 243(1308), 181-185. doi:10.1098/rspb.1991.0029

Gregory, T. R. (2001). Coincidence, coevolution, or causation? DNA Content, Cell Size, and the C-value Enigma. Biological Reviews, 76(1), 65-101. doi:10.1111/j.1469-185x.2000.tb00059.x

Hudson, M. E., Turner, A., \& Sewell, M. A. (2015). Comparative Ultrastructure of Spermatozoa from Two Regular and Two Irregular New Zealand Echinoids. Invertebrate Biology, 134(4), 341-351. doi:10.1111/ivb.12105

Immunofluorescence sample preparation. (n.d.). Retrieved June 21, 2016, from http://microscopy.duke.edu/sampleprep/if.html

Islam, M. S., \& Akhter, T. (2012). Tale of Fish Sperm and Factors Affecting Sperm Motility: A Review. Advances in Life Sciences, 1(1), 11-19. doi:10.5923/j. als. 20110101.03

Jamieson, B. G., \& Leung, L. K. (1991). Fish Evolution and Systematics: Evidence from Spermatozoa. Cambridge University Press. doi:10.1046/j.14209101.1992.5040721.x

Lamunyon, C. W., \& Ward, S. (2002). Evolution of Larger Sperm in Response to Experimentally Increased Sperm Competition in Caenorhabditis elegans. Proceedings of the Royal Society B: Biological Sciences, 269(1496), 1125 1128. doi:10.1098/rspb.2002.1996

Landry, C., Geyer, L. B., Arakaki, Y., Uehara, T., \& Palumbi, S. R. (2003). Recent Speciation in the Indo-West Pacific: Rapid Evolution of Gamete Recognition and Sperm Morphology in Cryptic Species of Sea Urchin. Proceedings of the Royal Society B: Biological Sciences, 270(1526), 1839-1847. doi:10.1098/rspb.2003.2395

Lessios, H. A., \& Cunningham, C. W. (1990). Gametic Incompatibility between Species of the Sea Urchin echinometra on the Two Sides of the Isthmus of Panama. Evolution, 44(4), 933-941. doi:10.2307/2409556

Life Technologies. Alexa Fluor ${ }^{\circledR} 488$ goat anti mouse IgG. Eugene, OR.

Life Technologies. Anti alpha tubulin (bovine), mouse IgG1, monoclonal 23610501. Eugene, OR.

Life Technologies. DAPI (4', 6-diamidino-2-phenylindole, d hydrochloride). Eugene, OR.

Marks, J. A., Biermann, C. H., Eanes, W. F., \& Kryvi, H. (2008). Sperm Polymorphism Within the Sea Urchin Strongylocentrotus droebachiensis: Divergence Between Pacific and Atlantic Oceans. The Biological Bulletin, 215(2), 115125. doi: $10.2307 / 25470692$

Mendelson, T. C., Imhoff, V. E. and Venditti, J. J. (2007). The Accumulation of Reproductive Barriers during Speciation: Postmating Barriers in Two Behaviorally Isolated Species of Darters (Percidae: Etheostoma). Evolution, 61: 2596-2606. doi: 10.1111/j.1558-5646.2007.00220.x

Montoto, L. G., Sanchez, M. V., Tourmente, M., Martin-Coello, J., Luque-Larena, J. J., Gomendio, M., \& Roldan, E. R. (2011). Sperm Competition Differentially Affects Swimming Velocity and Size of Spermatozoa from Closely Related Muroid Rodents: Head First. Reproduction, 142(6), 819-830. doi: $10.1530 /$ rep-11-0232

Parker, G. A. (1970). Sperm Competition and Its Evolutionary Consequences in the Insects. Biological Reviews, 45(4), 525-567. doi:10.1111/j.1469185x.1970.tb01176.x

Robinson, J. P., Sturgis, J., \& Kumar, G. L. (2009). Immunofluorescence. In G. L. Kumar \& L. Rudbeck (Eds.), IHC Staining Methods (5th ed., pp. 61-65). Carpinteria, CA: Dako North America.

StatCrunch [Computer software]. (2016). Retrieved from https://www.statcrunch. com/app/index.php?

Tanabe, T., \& Sota, T. (2008). Complex Copulatory Behavior and the Proximate 
Effect of Genital and Body Size Differences on Mechanical Reproductive Isolation in the Millipede Genus Parafontaria. The American Naturalist, 171(5), 692-699. doi:10.2307/30119668

Tarnowski, B. I., Spinale, F. G., \& Nicholson, J. H. (1991). DAPI as a Useful Stain for Nuclear Quantitation. Biotechnic \& Histochemistry, 66(6), 296-302. doi:10.3109/10520299109109990

Williams, T. H., \& Mendelson, T. C. (2010). Behavioral Isolation Based on Visual Signals in a Sympatric Pair of Darter Species. Ethology, 116(11), 1038-1049. doi:10.1111/j.1439-0310.2010.01816.x

Williams, T.H., \& Mendelson, T.C. (2014). Quantifying Reproductive Barriers in a Sympatric Pair of Darter Species. Evolutionary Biology, 41(2), $212-$ 220. doi: 10.1007/s11692-013-9259-y

Yániz, J., Palacín, I., Vicente-Fiel, S., Sánchez-Nadal, J., \& Santolaria, P. (2015). Sperm Population Structure in High and Low Field Fertility Rams. Animal Reproduction Science, 156, 128-134. doi:10.1016/j.anireprosci.2015.03.012 